\title{
Some Consequences of Thermosolutal Convection: The Grain Structure of Castings
}

\author{
G. HANSEN, A. HELLAWELL, S.Z. LU, and R.S. STEUBE
}

The essential principles of thermosolutal convection are outlined, and how convection provides a transport mechanism between the mushy region of a casting and the open bulk liquid is illustrated. The convective flow patterns which develop assist in heat exchange and macroscopic solute segregation during solidification; they also provide a mechanism for the transport of dendritic fragments from the mushy region into the bulk liquid. Surviving fragments become nuclei for equiaxed grains and so lead to blocking of the parental columnar, dendritic growth front from which they originated. The physical steps in such a sequence are considered and some experimental data are provided to support the argument.

\section{INTRODUCTION}

THE focus of this article is upon the grain structure of castings and whether it is possible to model the columnarequiaxed grain transition in a physically realistic way. The argument is that if no heterogeneous substrate materials (e.g., grain refiners) are deliberately introduced into a melt prior to or during pouring, the most probable alternative nuclei for equiaxed grains are particles of the base material itself. Such crystal fragments may be introduced during the pouring operation, especially with low superheats or, at longer times, can originate during dendrite ripening within the developing columnar mushy region. Without forced stirring, the only mechanism for such fragments to "escape" from the mushy region is by natural convectionso-called thermosolutal convection.

There is nothing new in this argument: it was suggested by Rosenhain nearly 70 years ago, ${ }^{[1]}$ and although it has been reiterated frequently, ${ }^{[2-6]}$ the details of how it might operate have been addressed only in a fragmentary way. An attempt will be made here to identify the physical steps which are implied by this process and to consider how they can be linked to provide a predictive sequence for equiaxed grain formation and, hence, for the columnar-to-equiaxed grain transition. It is in order, first, to outline the characteristics of thermosolutal convection, which is an essential part of the process.

\section{THERMOSOLUTAL CONVECTION}

Three statements, (1) through (3), summarize this phenomenon. ${ }^{[7, \mathbf{8}, \mathbf{9}]}$

1. Natural convection occurs because of density variations in a system. In a multicomponent system, density differences arise from temperature and compositional var-

G.C. HANSEN, Graduate Student, A. HELLAWELL, Professor, S.Z. LU, Research Associate Professor, and R.S. STEUBE, Graduate Student, are with the Metallurgical and Materials Engineering Department, Michigan Technological University, Houghton, MI 49931.

This article is based on a presentation made at the "Analysis and Modeling of Solidification" symposium as part of the 1994 Fall meeting of TMS in Rosemont, Illinois, October 2-6, 1994, under the auspices of the TMS Solidification Committee. iations which develop simultaneously. There are therefore two contributions to the buoyancy within a fluid:

(a) Thermo:

$$
\rho_{T}=\rho_{0}\left[1-\alpha\left(T-T_{0}\right)\right]
$$

where $\rho$ is density, $T$ is temperature, $\alpha$ is the volume coefficient of thermal expansion, which is always positive, and $\rho_{0}$ is the density at the melting point, $T_{0}$.

(b) Solutal:

$$
\rho_{C}=\rho_{0}\left[1 \pm \beta\left(C-C_{0}\right)\right]
$$

where the solutal expansion coefficient, $\beta$, can be of either sign and $\rho_{0}$ is the density of pure solvent liquid, as described previously.

Combining these, the density difference between two elements of liquid, $\Delta \rho$, having temperature and composition differences, $\Delta T$ and $\Delta C$, is then given by

$$
\Delta \rho=-\rho_{0}(\alpha \Delta T \pm \beta \Delta C)
$$

or is expressed as a vertical gradient,

$$
\frac{d \rho}{d z}=-\rho_{0}\left(\alpha \frac{d T}{d z} \pm \beta \frac{d C}{d z}\right)
$$

Of particular interest will be situations where the signs of $\alpha \Delta T$ and $\beta \Delta C$ are opposite, so that it becomes possible to have liquid which is both cooler and less dense. In the present context, the relevant compositions and temperatures are along the liquidus boundary of a phase diagram.

(2) If an element of liquid translates vertically from one position to another in a gravitational field at a velocity $V$ (Figure 1), because of an initial density difference with respect to surroundings, continued movement will depend upon there being retained buoyancy to sustain convection and therefore upon how rapidly heat and matter exchange laterally between the volume element and surroundings. With reference to Figure 1 , there are two fluxes.

(a) Thermo:

$$
J_{T}=k \frac{d T}{d x}=\kappa \frac{d Q}{d x}
$$

where $k$ and $\kappa$ are the thermal conductivity and diffusivity and $T$ and $Q$ are temperature and heat content, respectively 


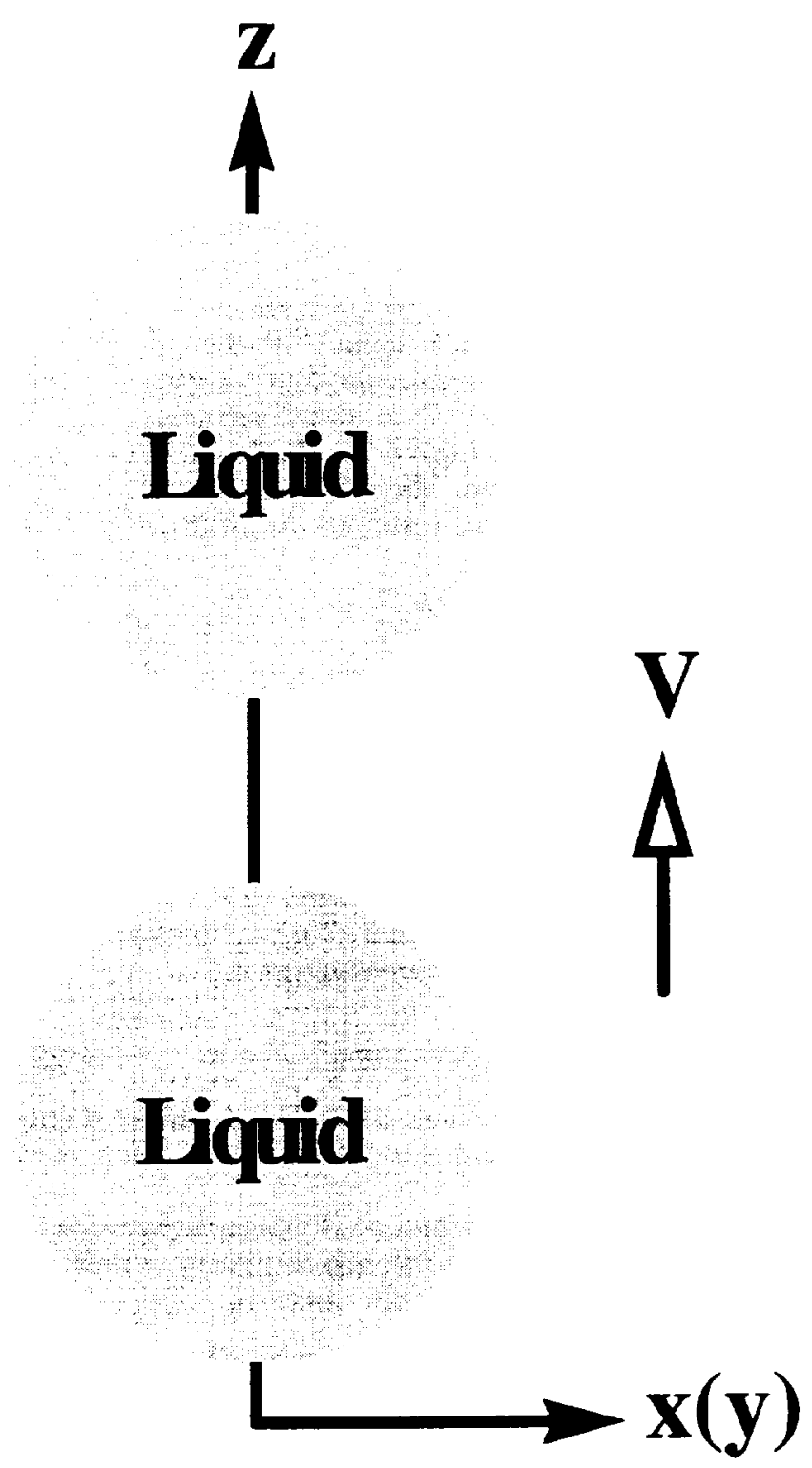

Fig. 1-Schematic for Egs. [3a] and [b] for a volume element of liquid rising at a rate $V$.

( $\kappa=k / C p$, where $C p$ is the specific heat at constant pressure); $Q$ and $C p$ are here defined with respect to volume. (b) Solutal:

$$
J_{C}=D_{L} \frac{d C}{d x}
$$

where $D_{L}$ is the liquid diffusion coefficient and $C$ is concentration.

Of importance here is the ratio of the two diffusivity coefficients:

$$
\frac{\kappa}{D_{L}}=\tau
$$

where $\tau$ is called the Lewis number, having values $\approx 10^{3}$ for metals or $\approx 10^{2}$ for aqueous and organic systems. Heat redistribution is therefore rapid and the buoyancy is sustained by a composition difference of much longer duration. (3) Fluid flow is opposed by viscous drag and a velocity gradient is set up around a moving element as it shears through the surrounding liquid. If the flow is streamlined, as it generally is in the cases considered here, the buoyancy pressure is related to the velocity gradient by the dynamic viscosity coefficient, $\eta$ :

$$
\Delta \rho g h=\eta \frac{d v}{d x}
$$

more conveniently normalized as the kinematic viscosity coefficient, $\nu=\frac{\eta}{\rho}$.

It is the combination of these three relations that determines the scale of convective patterns and rate of flow within them. As a general statement, for such convection to occur, the diffusivity ratio cannot equal that of the buoyancy contributions; i.e.,

$$
\tau=\frac{\kappa}{D_{L}} \neq \frac{\alpha(d T / d x)}{\beta(d c / d x)}
$$

and, in general, $\tau$ exceeds that ratio by some two orders of magnitude.

In fluid mechanical calculations, it is mathernatically convenient to describe different situations or problems by dimensionless numbers, relating system parameters, velocity $V\left(L T^{-1}\right)$, and scale $(L)$ of events to relevant material constants, notably, $\kappa, D_{L}$, and $\nu\left(L^{2} T^{-1}\right)$. A few of these numbers are listed in Table I, with indications of their physical meanings and orders of magnitude relevant to the present context. Clearly, the values of those numbers involving system parameters are particularly sensitive to the choice of that dimension, $L$, describing the scale of convective flow, e.g., height or width of the system or of the local flow pattern. Since, in the present context we are concerned with local flow plumes of the order of $10^{-3} \mathrm{~m}$ wide (but much longer), the numbers quoted are for that scale. We note in particular that the Reynolds number, even for metallic systems, is below the limit $\left(\approx 10^{3}\right)$ at which streamlined flow breaks down into turbulence. It will also be apparent that Péclet numbers, $\mathrm{Pe}_{T}$ or $\mathrm{Pe}_{s}$ are products of the Reynolds number and the Prandtl or Schmidt number. Keeping the physical significance of these numbers in perspective is crucial to understand their implications. Thus, referring to the Péclet numbers, a value of one would indicate that heat or mass transfer took place as rapidly as convective flow, so that the fluid would remain quiescent. In the present context, the two Péclet numbers indicate that fluid flow typically takes place a little faster than allows thermal equilibrium to be attained, but very much faster than compositional (solutal) adjustment, cf Eqs. [3] and [4]. The relatively big difference between Prandtl numbers for metals $v s$ aqueous and organic systems reflects their higher thermal conductivity, but as it transpires, this does not significantly affect the scale of convective patterns, although the flow rates are much rapid.

\section{PHENOMENOLOGICAL CONSEQUENCES}

Figure 2 shows three schematic casting configurations and the form of thermosolutal flow patterns which are to be expected or are observed. The left-hand sequence, I through III (a), corresponds to $d \rho / d z$ negative, i.e., solute 
Table I. To Identify Relevant Dimensionless Numbers

\begin{tabular}{|c|c|c|c|}
\hline Name & Ratio & Physical Meaning & Order of Magnitude \\
\hline Prandtl & $\sigma=\nu / \kappa$ & velocity vs temperature field & $\sim 10=$ (metals) to $\sim 10$ (aqueous, organic) \\
\hline Schmidt & $\mathrm{SC}=\nu / D$ & velocity vs composition field & $\sim 10^{2}$ (metals) to $\sim 10^{3}$ (aqueous, organic) \\
\hline Reynolds & $\mathrm{RE}=\frac{V L}{\nu}$ & convective vs diffusive momentum transfer & $-5 \times 10^{2}$ (metals to $\leq 10^{2}$ (aqueous organic) \\
\hline Péclet & $\begin{aligned} \mathrm{Pe}_{\tau} & =\frac{V L}{\kappa} \\
\mathrm{Pe}_{s} & =\frac{V L}{D}\end{aligned}$ & $\begin{array}{l}\text { convective vs thermal transfer } \\
\text { convective vs solutal transfer }\end{array}$ & $\begin{array}{c}-10 \\
\sim 10^{4} \text { (metals) to } \sim 10^{3} \text { (aqueous, organic) }\end{array}$ \\
\hline
\end{tabular}

sufficiently less dense to outweigh the thermal buoyancy (cooler liquid is less dense), and the right-hand sequence, I through III (b), is the reverse, with more dense solute. These would correspond to lead base alloys with tin and vice versa. The shaded regions correspond to columnar mushy regions, growing into positive temperature gradients with the gravitational vector vertically downward. The arrows, $V$, indicate the directions of growth. Cases $\mathrm{I}(\mathrm{a})$ and (b), with growth vertically upward, have the supernatant liquid stabilized against convection by the positive temperature gradient, but there is density inversion in case I(a) because the cooler interdendritic liquid is less dense; this will be of central interest hereafter. In case I(b), the entire liquid is quiescent; $d \rho / d z$ is negative. In cases II(a) and (b), the open liquid will be mixed by thermally driven convection; in case $\Pi(a)$, the less dense interdendritic liquid will not contribute to convection, but in case II(b), the solutal contribution will tend to cause downward flow from the growth front, interacting with thermal convection. In cases III(a) and (b), with solidification from the sides, solutal buoyancy will cause (a) upward or (b) downward convection at and behind the growth fronts, and these will also interact with thermal convection which is not here represented. Macroscopic solute segregation in these two cases will typically reduce the freezing point at the (a) top or (b) bottom of the ingots, so that the upper and lower parts of the growth fronts present lopsided versions of I(a) or II(b).

We will concentrate on the configuration of I(a), because it allows the thermal and solutal contributions to be most easily separated and is thus the most analytically tractable of these alternatives. References to the other configurations will be made occasionally, but in any case, extensions to these can easily be anticipated in a qualitative way.

Referring then to case I(a) (Figure 3), from left to right: (a) alloy of composition $C_{0}$ in a phase diagram with $k_{0}<$ 1 and negative liquidus slope, $m_{L}$, grows upward with liquid concentration rising to $C$, at dendrite tips; (b) there is a concentration ripple across the front of the wave length determined by the primary dendritic spacing; (c) concentration profiles along the vertical axes $A$ and $B$ then appear, with interdendritic liquid, trace $B$, rising according to the reciprocal liquidus slope and vertical temperature gradient, i.e., $d C / d z=d C / d T \cdot d T / d z$; and (d) the corresponding vertical density profiles showing, with $\beta d C / d z>-\alpha d T / d z$ (i.e., Eq. [2b]), a small dip at dendrite tips, corresponding to $C_{l}$, while the interdendritic liquid shows density inversion with a positive value of $d \rho i d z$. (If the solutal coefficient $\beta=0$, the density profile would continue downward ac-

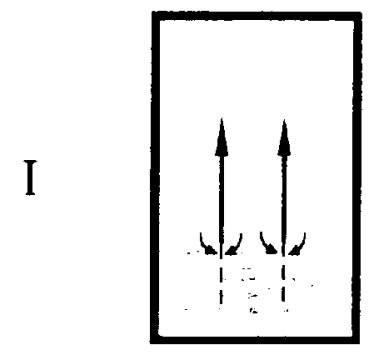

$\Delta \rho<0$

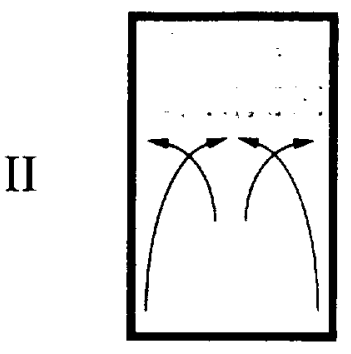

$\Delta \rho<0$

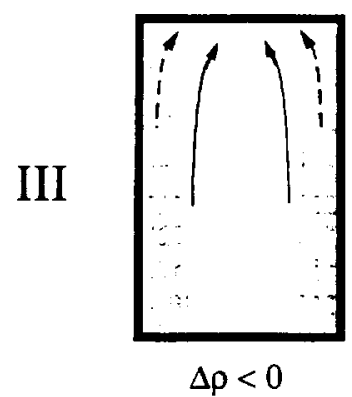

(a)

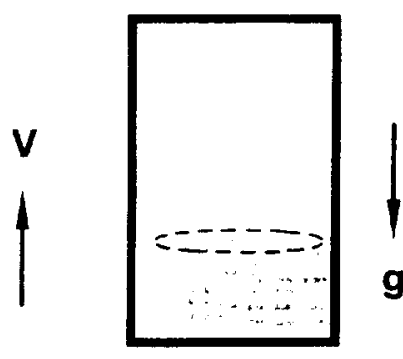

$\Delta p>0$

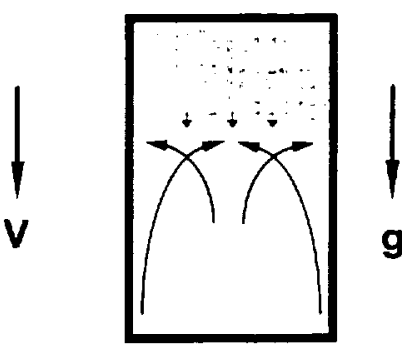

$\Delta \rho>0$
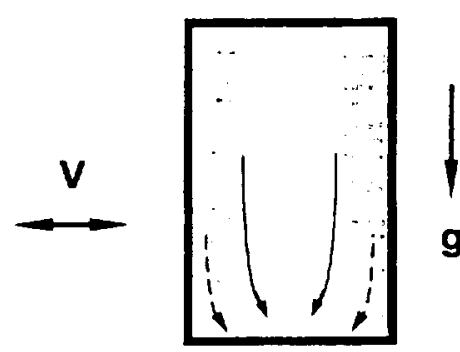

$\Delta \rho>0$

(b)
Fig. 2-Altemative casting geometries, I through III, with schematic thermosolutal convection patterns, solid lines within open liquid, broken lines; interdendritic:shaded regions correspond to permeable mushy regions: $(a)$ for cases where the solute is less dense than the solvent, $\beta d C / d z>-\alpha d T / d z$ (cooler liquid is the less dense) and $(b)$ for the reverse situation where the thermosolutal buoyancy terms have the same sign (solute is the more dense). The arrows, $V$, indicate the growth directions: I-upward, II-downward, and III-from the sides, horizontally. The gravitational vector, $g$, is vertically downward in all cases.

cording to the temperature gradient.) With such density inversion, the system is unstable and thermosolutal convection is expected. The critical region for the perturbation(s) leading to such convection is close to the growth 


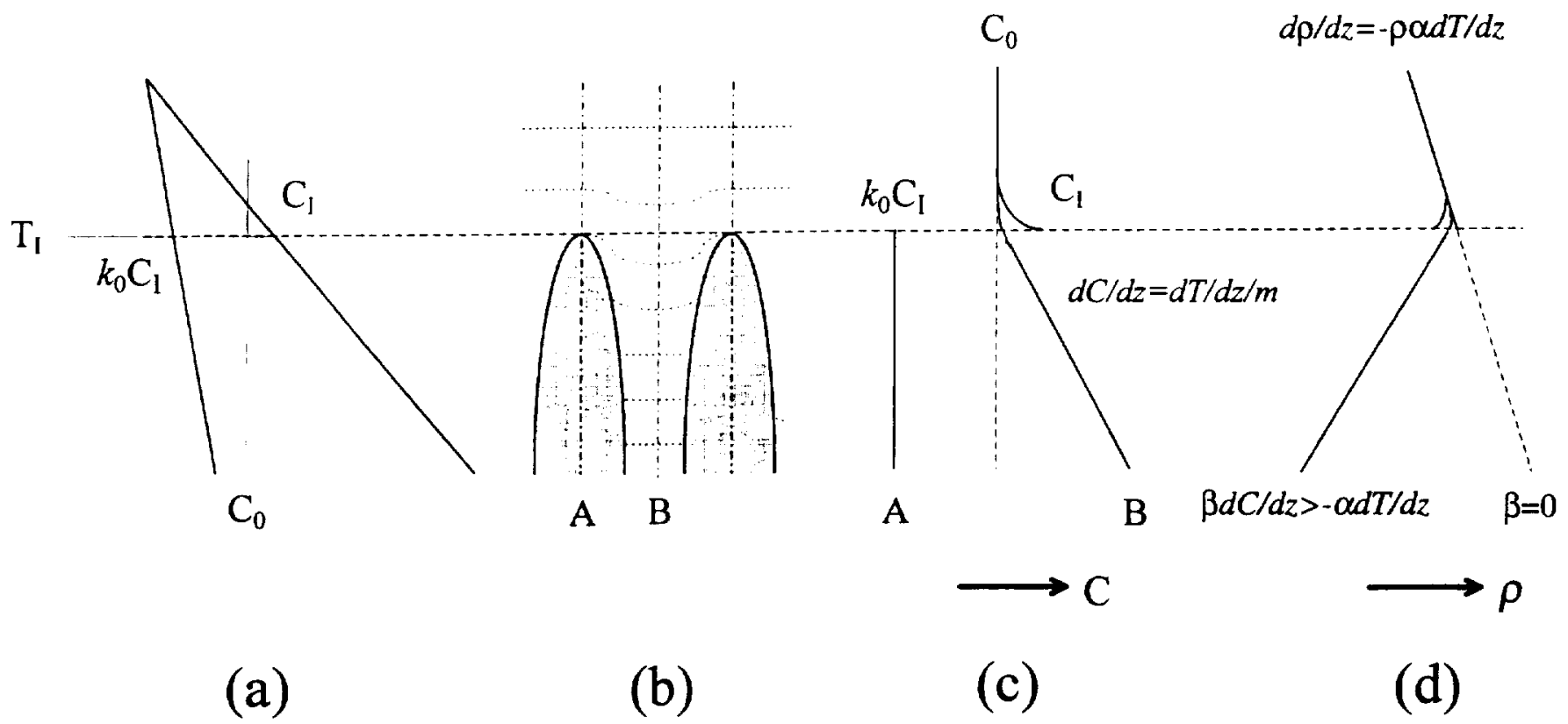

Fig. 3-(c) Composition and $(d)$ density profiles through $(b)$ an upward growing dendritic front. (a) Trace A along a dendrite axis and trace B, interdendritic, with reference to a phase diagram and alloy composition $C$.

front where the density gradient changes sign, and experimentation confirms that this is so. ${ }^{[12,13]}$ Perturbations at lower positions within the mushy region are improbable. The consequences of this configuration are well documented for transparent systems ${ }^{[0-15]}$ and are briefly as follows: very early $\left(<10^{2} \mathrm{~s}\right)$ in the growth of the dendritic front, short-range convective "fingers" develop, rising up to $\approx 10 \mathrm{~mm}$ into the supernatant liquid with concomitant downward flow to create a recirculating, mixing zone, which is slightly solute enriched, approximately 1 to 2 pct. At somewhat longer times $\left(>10^{3} \mathrm{~s}\right)$, finger convection couples with the interdendritic liquid and locally reinforces to produce longer range $(>100 \mathrm{~mm})$ solute-rich plumes. As these develop, the shorter range patterns are largely replaced and, simultaneously with plume formation, channels develop within the dendritic array below. These features can be observed directly in a transparent aqueous or organic system and Figures 4 (a) through (c) are examples from the aqueous ammonium chloride water system, in which water is the less dense solute. Figure $4(d)$ is an example of such channel formation in a metallic ingot, where accumulation of dendritic debris within channels gives rise to characteristic polycrystalline freckles which delineate the original events. ${ }^{[15]}$ In Figure 4(a) is shown the short-range, oscillatory finger convection patterns, while Figure $4(\mathrm{~b})$ shows the longer range, nearly steady state plumes, which rise from channel mouths, such as that shown in Figure $4(\mathrm{c})$. The dimensions of channels and dendrites are very similar in aqueous and metallic systems, although the flow rates in the latter are undoubtedly much faster. ${ }^{[15]}$

Measurement and analysis of channel-plume motion in transparent systems allows extrapolation to opaque metallic systems, based on postmortem examination of the fully solidified samples. ${ }^{[14.15]}$ Plume widths are all about $10^{-3} \mathrm{~m}$ (some three to five primary dendrite spacings), and plumes are enriched in solute by 1 to 2 pct compared with the bulk liquid; as liquid leaves the dendritic front, it is also a little cooler $(<5 \mathrm{~K})$ than the surroundings but rapidly warms up to the ambient temperature ( $c f$ thermal Péclet number). Flow rates in the plumes have been measured in the transparent materials and analysis of these, extrapolated to metals, indicates mean velocities, organic:aqueous:metallic, of about $10^{-3} \mathrm{~ms}^{-1}: 10^{-2} \mathrm{~ms}^{-1}: 10^{-1} \mathrm{~ms}^{-1}$, the last being very rapid but still streamlined. At a given time, some 2 to 3 pct of the liquid flows upward rapidly in such plumes, while the balance is drawn down into the upper part $(<10 \mathrm{~mm}$ ) of the mushy region to be continuously entrained in the plumes. This means, as shown in Figure 4(c), that solventrich, warmer liquid is drawn through the upper part of the dendritic array. The finger convection also involves some short-range recirculation between dendrites, but it does not penetrate the mushy region to greater depths than the primary spacing $\left(<10^{-3} \mathrm{~m}\right)$ and there are no channels associated with it. It may be noted that in a configuration with horizontal growth, as in a billet casting, Figure 2, III, the finger zone can never be fully established but is mostly swept away by upward or downward flow across the growth front. Plume flow is similarly obscured at such a front, but this does not mean that exchange between the mushy region and open liquid is absent.

In all cases, the convection currents carry entrained dendritic fragments within them and thus provide particle transport over shorter or longer ranges into the bulk liquid. This will be our principal focus.

It should be added here that there are two other consequences of this convection, namely, macroscopic thermal and solutal mixing within the open bulk liquid. These are particularly obvious in the vertical configuration of Figure 2, $\mathrm{I}(\mathrm{a})$. The re-entrainment means that there is accelerated heat exchange between the open liquid and the mushy region, causing the former to cool more rapidly than it would by diffusion alone. Simultaneously, liquid mixing causes macrosegregation and progressive depression of the freezing point, so that the schematic concentration profile of Fig- 


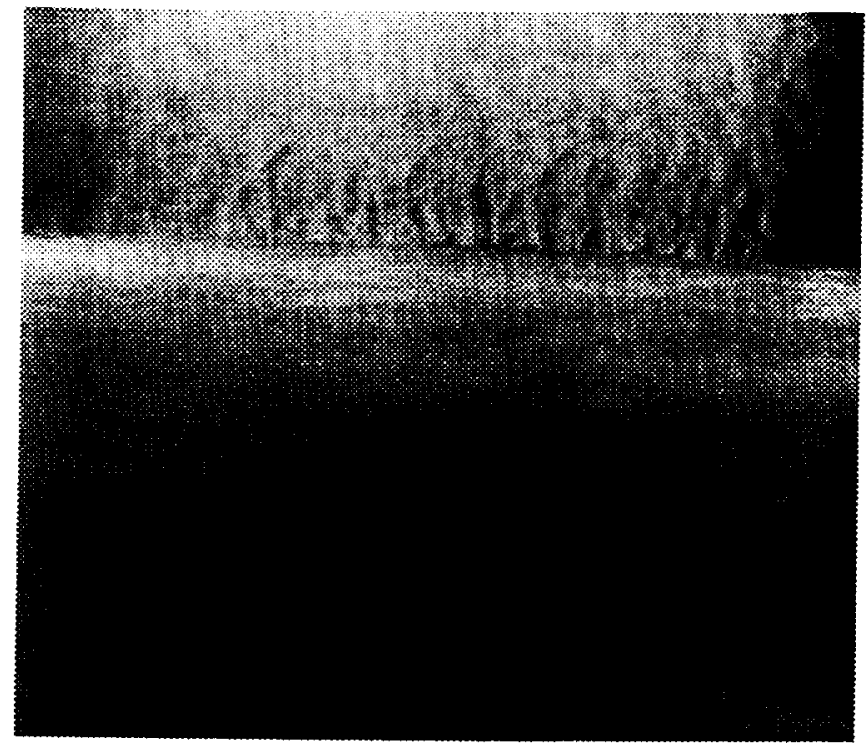

$1 \mathrm{~cm}$

(a)

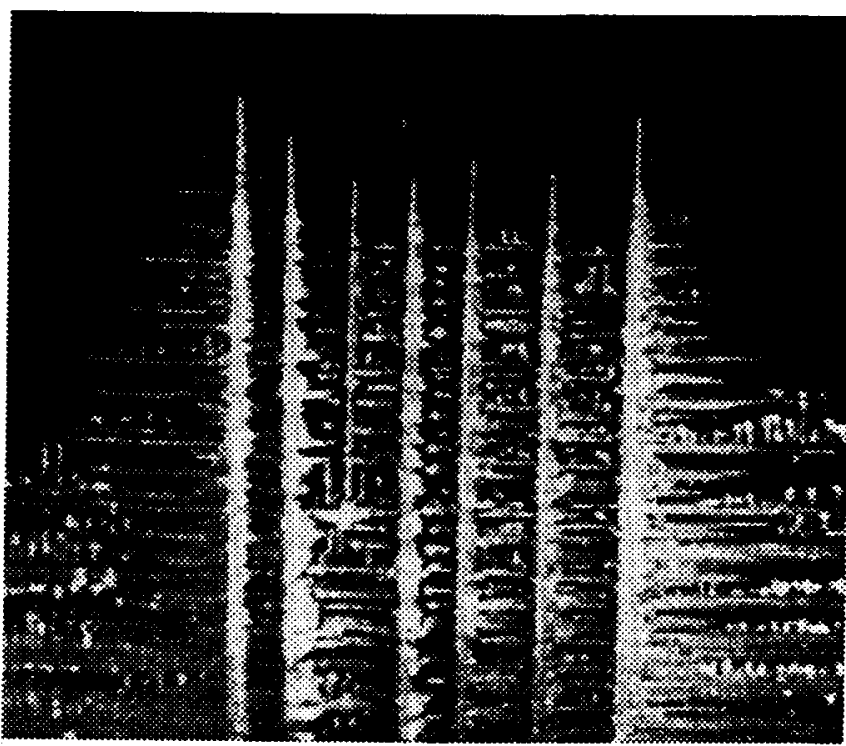

$1 \mathrm{~mm}$

(c)

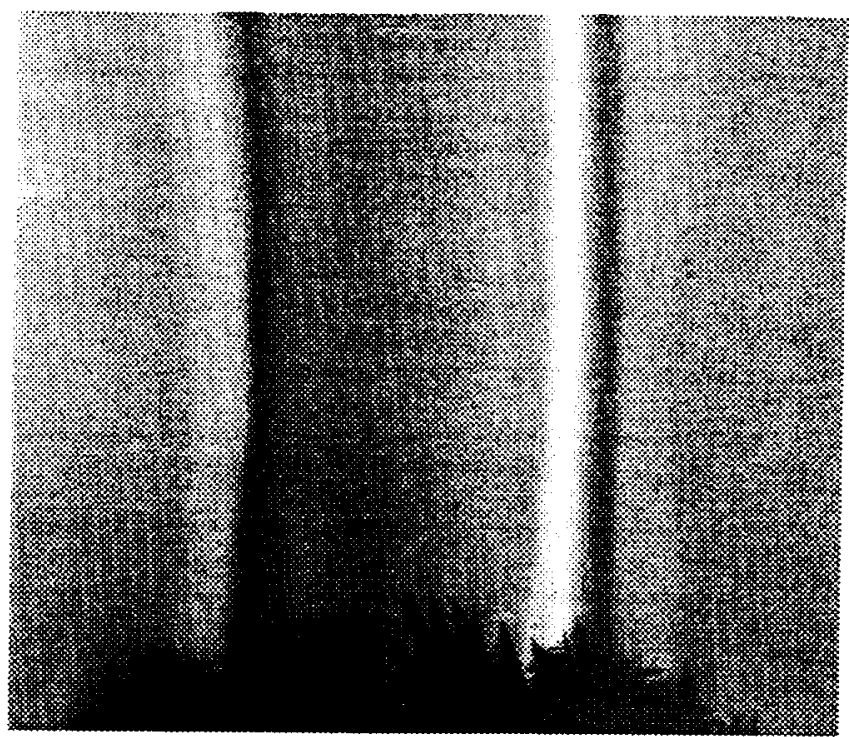

$1 \mathrm{~mm}$

(b)

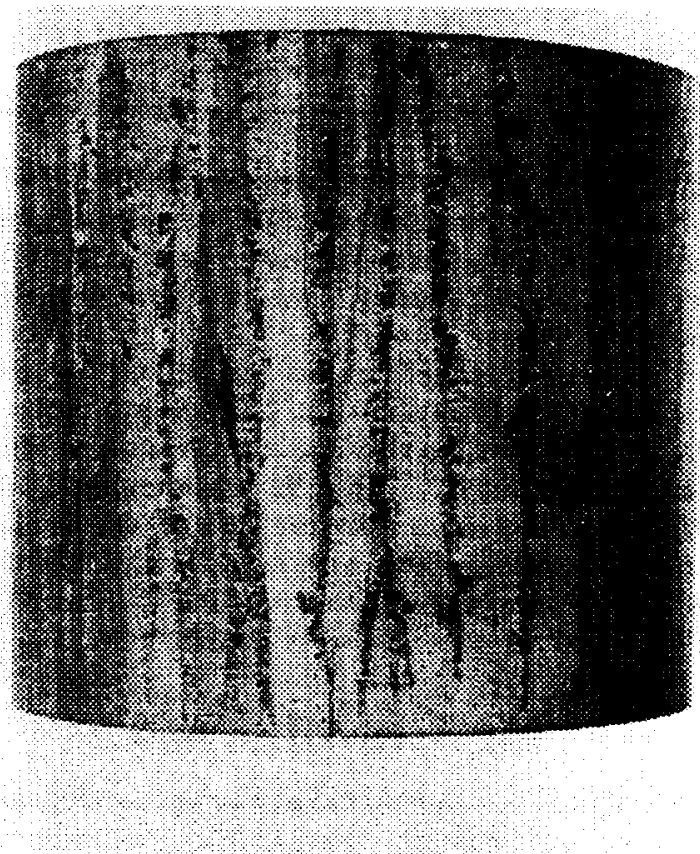

$3 \mathrm{~cm}$

(d)

Fig. 4 (a) Side view of short range finger convection above a dendritic growth front in $\mathrm{NH}_{4} \mathrm{Cl}-\mathrm{H}_{2} \mathrm{O}$; (b) long-range plume convection from channel mouths at a similar growth front. The optical contrast in both cases arises from composition-dependent variations in refractive index. (c) Detail of a channel mouth from which a convection plume, (b), is flowing, showing scale of channel and dendrites: and (d), surface freckles on the outside of a superalloy ingot (courtesy of Giamei, United Technologies Research Center, Dayton, OH). Scales as shown. All relate to case I(a) of Fig. 2.

ure $3(c)$ is not actually steady state, i.e., $C_{L} \neq C_{0}$. During the regime of finger convection, growth takes place into a solute-enriched zone, comparable with a zone-melting con- figuration. With longer range mixing of the liquid, the solute redistribution can be described by an effective distribution coefficient, $k_{\mathrm{eff}}$ where $k_{0}<k_{\mathrm{eff}}<1$. Without 
Table II. Model Sequence for Grain Structure (Events/ Volume/Time)

(1)

Side arm generation and ripening
$\left(>10^{6} \mathrm{~cm}^{-3}\right.$ at $\left.>10^{2} \mathrm{~cm}^{-2} \mathrm{~s}^{-1}\right)$

$\downarrow$

(2)

Side arm separation with and without fluid flow (fraction $>10^{-1}$ )

Fragment escape by convection/stirring
$\left(>10 \mathrm{~cm}^{-2} \mathrm{~s}^{-1}\right)$

$\downarrow$

(4)

Fragment survival
(melting/life span/population density)

$\downarrow$

(5)

\begin{tabular}{|c|}
\hline $\begin{array}{c}\text { Growth and sedimentation } \\
\text { (modified stokes) }\end{array}$ \\
$\downarrow$ \\
\hline $\begin{array}{c}\text { Columnar blocking and grain size } \\
\text { (columnar/equiaxed) }\end{array}$ \\
\hline
\end{tabular}

such liquid mixing, steady state prevails and $k_{\text {eff }}=1$; with mixing, $k_{\text {eff }}$ may be as low as 0.9 and the mean mushy zone composition is adjusted by a larger solid fraction than the lever rule would predict. This has been analyzed elsewhere. ${ }^{[15]}$

With these essential features in mind, we proceed to the question of modeling how the grain structure of a casting can develop when no relevant heterogeneous substrates are present in the liquid. This is the situation if deliberate additions have not been made to the melt and assumes that dendrite fragments of the base material must be better "nuclei" for equiaxed grains than any other accidental "dirt" in the system, such as oxide or slag particles.

\section{A PHYSICAL MODEL}

In Table II is a model to describe the development of an equiaxed grain structure from intrinsic nucleation sites involving sequential steps (1) through (6), as follows:

(1) Side arms of dendrites are continuously generated and coarsen by ripening behind the columnar growth front;

(2) A fraction of these side arms become detached from primary dendrite stalks;

(3) A further fraction escape from the mushy zone into the bulk liquid;

(4) Some of these survive, subject to time, local temperature, and composition;

(5) Survivors grow in supercooled liquid and sink as they do so,

(6) Survivors eventually block the advance of the parental growth front from which they originated.

The details could be further subdivided according to choice, but this is a closed system (any casting, even a
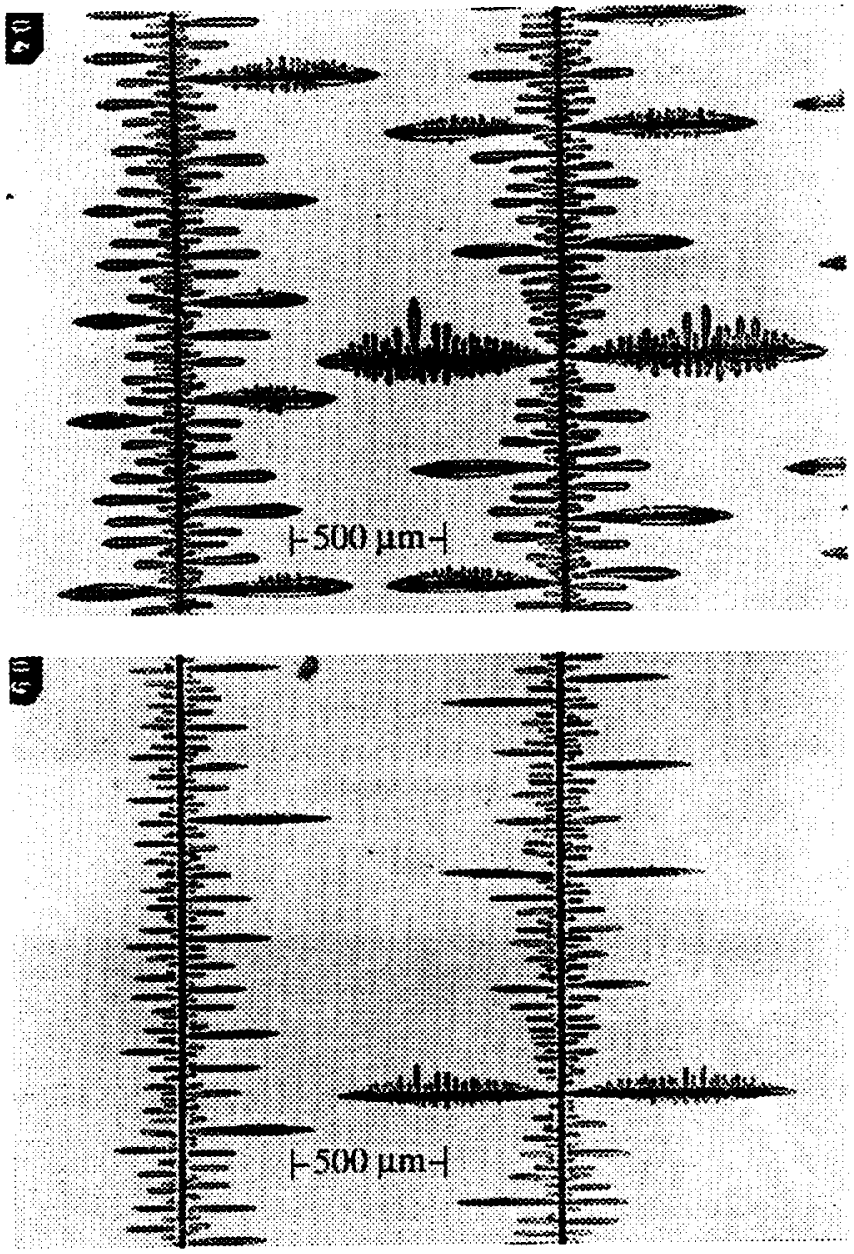

Fig. 5- $\mathrm{NH}_{4} \mathrm{Cl}$ dendrites growing horizontally on a gradient stage: (a) under steady-state conditions, with little, if any, secondary arm detachment; and (b) after a small temperature rise, when nearly all side arms become detached.

continuous casting, constitutes a closed system) and the sequence describes a physically realistic and observed situation. There are no nucleation kinetics in the classical sense, apart from those of a chilled layer on the mold walls, from which the columnar, dendritic front evolves. We consider these steps separately, indicating order of magnitude estimates where or when available, referring mostly to observations of the transparent system $\mathrm{NH}_{4} \mathrm{Cl}-\mathrm{H}_{2} \mathrm{O}$.

\section{A. Dendrite Structure}

Figure 5 shows $\mathrm{NH}_{4} \mathrm{Cl}$ dendrites growing horizontally in a thin cell $(\sim 125 \mu \mathrm{m})$ on a temperature gradient stage. There could be little or no convection in such a cell. The primary dendrite spacing is comparable with, or a little larger than, that observed in a casting of the same nominal composition. Many similar pictures have been shown in the literature, ${ }^{[4,16,17]}$ and we will make only a few observations. The density of side arms is very high and in a "typical" mushy region equates with some $10^{6} \mathrm{~cm}^{-3}$ within this region. The number of potential crystal fragments is enormous, even disregarding tertiary arms. With a growth rate of $\sim 10^{-3} \mathrm{~cm} \mathrm{~s}^{-1}$, side arms are continuously generated at a rate around $10^{3} \mathrm{~cm}^{-2} \mathrm{~s}^{-1}$ across the dendritic growth front. Side arms have initial, intrinsic, or nascent spacings, $\lambda_{2}^{o}$, 
which are in a simple ratio to the radii of curvature, $r$, of the primary dendrite tips and hence can be related to the imposed growth rate, composition, temperature gradient, and primary spacings, as analyses have shown. ${ }^{[18,23]}$ In this material, over an order of magnitude in the growth rate, this ratio is approximately $\lambda_{2}^{o}: r=4: 1$. We take this as a reflection of the oscillatory growth of dendrites, growing at the condition of "marginal stability," and believe that side arms grow from the initial perturbations so created at the primary tips. Discussion of the geometry, wave length, and frequency of dendrite tip perturbations is beyond the immediate scope of this article, but we note that side arm spacings are always in a simple ratio to the tip curvature, ${ }^{[19,20]}$ whether in single-component systems (heat flow only) or with solute present. Ripening of the intrinsic spacing begins almost at once and proceeds according to a relationship of the form $\lambda_{2}-\lambda_{2}^{0}=K t^{n}$, where $t=$ time and $n$ lies between $1 / 3$ and $1 / 2$ depending on the solid volume fraction, as described elsewhere in this series. ${ }^{[21]}$ This step is sufficiently well documented to be predictive.

\section{B. Side Arm Detachment}

It may be seen from Figure 5(a) that very few side arms have separated from the primary spine, although the junctions between them have necked down to small radii of curvature. This seems to be the situation if the temperature gradient is constant and the sample is cooling continuously. However, a small temperature rise (as yet undetermined) causes nearly all the side arms to detach abruptly (Figure $5(b)$ ), as Sato et al. ${ }^{[22]}$ also demonstrated with succinonitrile. In practice, such a temperature rise can occur if the mold temperature rises, as in a sand casting, or, of particular relevance, when the growth rate decreases, as it does continuously in the columnar region of a casting. With deceleration, there is an immediate increase in the dendrite tip radii, but a much slower increase in the primary spacings (e.g., Figure 7 of Reference 4). Consequently, the interdendritic solute concentration is raised above that for growth at the former growth rate and this causes side arms to melt off at their narrow roots. With such transient growth conditions, dendrites are always a little closer together then they would be under steady-state conditions. ${ }^{1401}$

Better temperature measurement and analysis of this situation are needed if there are to be predictive statements. We note that ripening, detachment, and re-entrainment take place continuously and concurrently and that the permeability of the dendritic array is also changing with position behind the growth front.

\section{Fragment Escape}

Reference to the transport of dendrite fragments by thermosolutal fingers and plumes has already been made. In the vertical configuration of Figure $2, \mathrm{I}(\mathrm{a})$, finger convection is of short range and involves only local recirculation. An estimate from video records would be around 10 particles $\mathrm{cm}^{-2} \mathrm{~s}^{-1}$ so ejected, having diameters between 10 to 40 $\mu \mathrm{m}$. In the vertical configuration, these may not be of great importance for future equiaxed grain formation, but in a casting with side chill, such currents are swept away into the open liquid, as already noted. Within plumes, fragments are more numerous and are traveling faster, but with the spatial separation of channels of $\approx 5 \mathrm{~mm}$, this again equates with a mean rate of escape around 10 to 30 fragments $\mathrm{cm}^{-2}$ $\mathrm{s}^{-1}$, against some 500 primary dendrites $\mathrm{cm}^{-2}$. With the regeneration of side arms, around $200 \mathrm{~cm}^{-2} \mathrm{~s}^{-1}$, previously noted, this must mean that about 10 pct of the potentially available fragments leave the mushy region and therefore that the fraction detached must be greater than this, but we do not yet know by how much. For the present, in the aqueous system, it will be safe to assume a particle escape density of $>10 \mathrm{~cm}^{-2} \mathrm{~s}^{-1}$, with a lower size estimate limited by resolution to $\approx 10 \mu \mathrm{m}$ or, on average, $\approx 20$ - $\mu \mathrm{m}$ diameter. There can be no estimates for a metallic system, as yet. Flow rates in metals should be an order of magnitude greater within plumes of similar diameters and spacings. ${ }^{[15]}$ We are limited here by uncertainty about the detachment kinetics.

\section{Fragment Survival}

Three areas, (a) through (c), may be identified:

(a) To treat the problem of survival requires a detailed thermal record with respect to time and position, in this case, as vertical temperature profiles, against which are required the corresponding particle population densities. Schematically (Figure 6(a)) the bulk liquid is cooling by heat extraction from the chilled base, assisted by convective mixing and heat exchange with the mushy region during re-entrainment. The temperature profiles are hinged about the growth front at $T_{t}$, so that predictive analysis of the interfacial undercooling, $\Delta T_{b}$, is an essential part of this problem ( $c f$ References 18 and 23). Temperature gradients in the liquid rapidly decrease with time, especially in metals and with convective mixing, so that in a very short time interval (10 to $10^{2} \mathrm{~s}$ ), there is a transition from most of the liquid being slightly superheated to its being almost uniformly supercooled. Above the freezing point, fragments melt, but as the profile falls below $T_{L}$, all survivors start to grow, recalescence occurs, and the liquid undercooling becomes small and uniform. With knowledge of the material properties (conductivities, specific, and latent heats), the liquid composition and, with a model for undercooling at the growth front, the temperature profiles can be computed with readily available thermal codes. This is a simple one-dimensional analysis which, however, must also incorporate thermal mixing in the open liquid by convection and the attendant heat exchange during re-entrainment. Experimentally, the temperatures can also be followed by a series of thermocouples or, better, can be scanned by one thermocouple, moving vertically up and down through the melt. Figure 6(b) is an example of such a scanning sequence for an aqueous $\mathrm{NH}_{4} \mathrm{Cl}$ casting, poured into a mold with an initial superheat of $\approx 30 \mathrm{~K}$ above the liquidus temperature. The positions and temperatures of the growth front are indicated on these temperature profiles and reflect the depression of the growth front from changes in bulk liquid composition (the finger zone dominates up to a time of about 30 minutes, after which macrosegregation occurs) and from the front undercooling, $\Delta T_{t} \approx 3$ to $4 \mathrm{~K}$.

(b) Until the bulk liquid falls below the freezing point, ejected particles melt and have a limited life span. The 


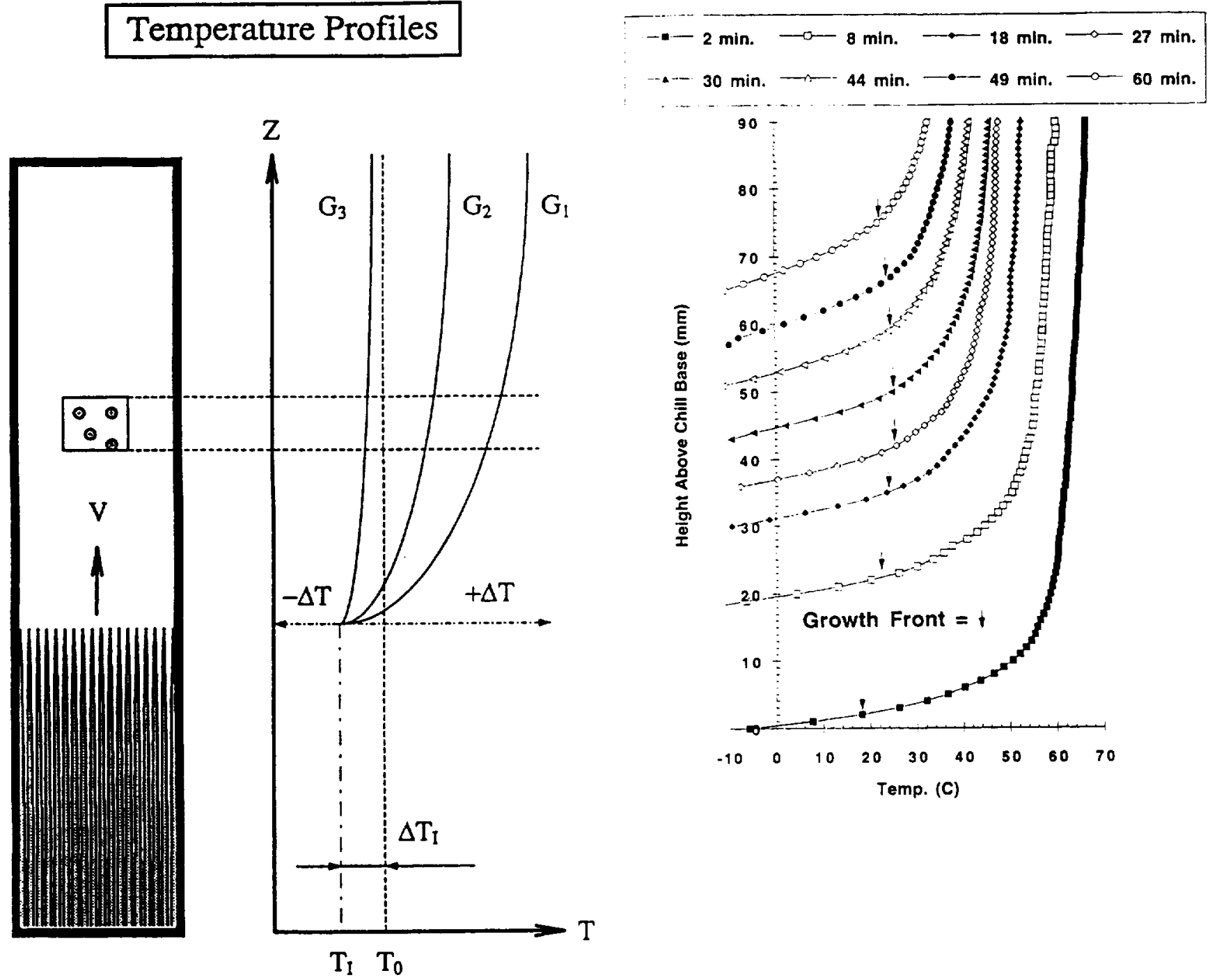

Fig. $6-(a)$ Schematic vertical temperature profiles, $G_{1}$ through $G_{3}$, at successively later times, above a dendritic front, growing at an undercooling, $\Delta T_{h}$ for velocity, $V$, and $(b)$ actual temperature profiles recorded by a single thermocouple, scanning vertically through an $\mathrm{NH}_{4} \mathrm{Cl}_{-} \mathrm{H}_{2} \mathrm{O}$ casting (positions of the growth front are shown by arrows).

kinetics of melting depend upon the ambient temperature, composition, and rate of movement of the particle through the liquid. In the present context, the total mass of solid is very small by comparison with the volume of liquid, so that the latent heat required for melting and the composition changes resulting therefrom are negligible. The problem may therefore be described as one of "unconstrained" melting. The rate of melting will clearly be a sensitive function of movement with respect to the surroundings, whether by long-range diffusion (of heat or matter) or through a boundary layer of limited thickness, but initially, it is necessary to separate the contributions from heat and matter transport. It will be assumed here that the times involved are small and that solid-state diffusion is not important. Experimental observations of ammonium chloride particles in aqueous castings indicate that in a thermally hostile environment, fragments of $10-$ to $20-\mu \mathrm{m}$ diameter rarely survive longer than $\approx 10$ seconds. The term "hostile," of course, needs qualification, because in the finger regime, there is continuous mixing of cooler, water-rich liquid with warmer salt-rich liquid, and plume temperatures are initially below those of the bulk liquid, so the precise environment is difficult to specify. However, reference to the schematic phase diagram of Figure 7 will show that there must be a transition in melting kinetics from a regime controlled by matter transport to one dominated by heat flow.

Consider (Figure 7), a bath composition of $C_{0}$ on a phase diagram of given liquidus slope (solid line of slope $m_{L}=$ $-\Delta T / \Delta C$ ). In the first place, consider a system such as $\mathrm{NH}_{4} \mathrm{Cl}-\mathrm{H}_{2} \mathrm{O}$ having zero solid solubility, $k_{0}=0$. A particle, initially at equilibrium at $T_{0}$, is placed in a large volume of liquid, $C_{0}$, at a superheat of $\Delta T_{h}$, where the equilibrium solute concentration is lower, by $\Delta C$. Melting is then at a rate $V_{m}$, given by

$$
V_{m}\left(C_{I}-C_{S}\right)=\frac{D_{L} \Delta C}{\Delta r}
$$

where $C_{l}$ is the liquid interfacial composition at $T_{0}+\Delta T_{h}$, $C$, is the solid composition $=0, D_{L}$ is the liquid diffusion 


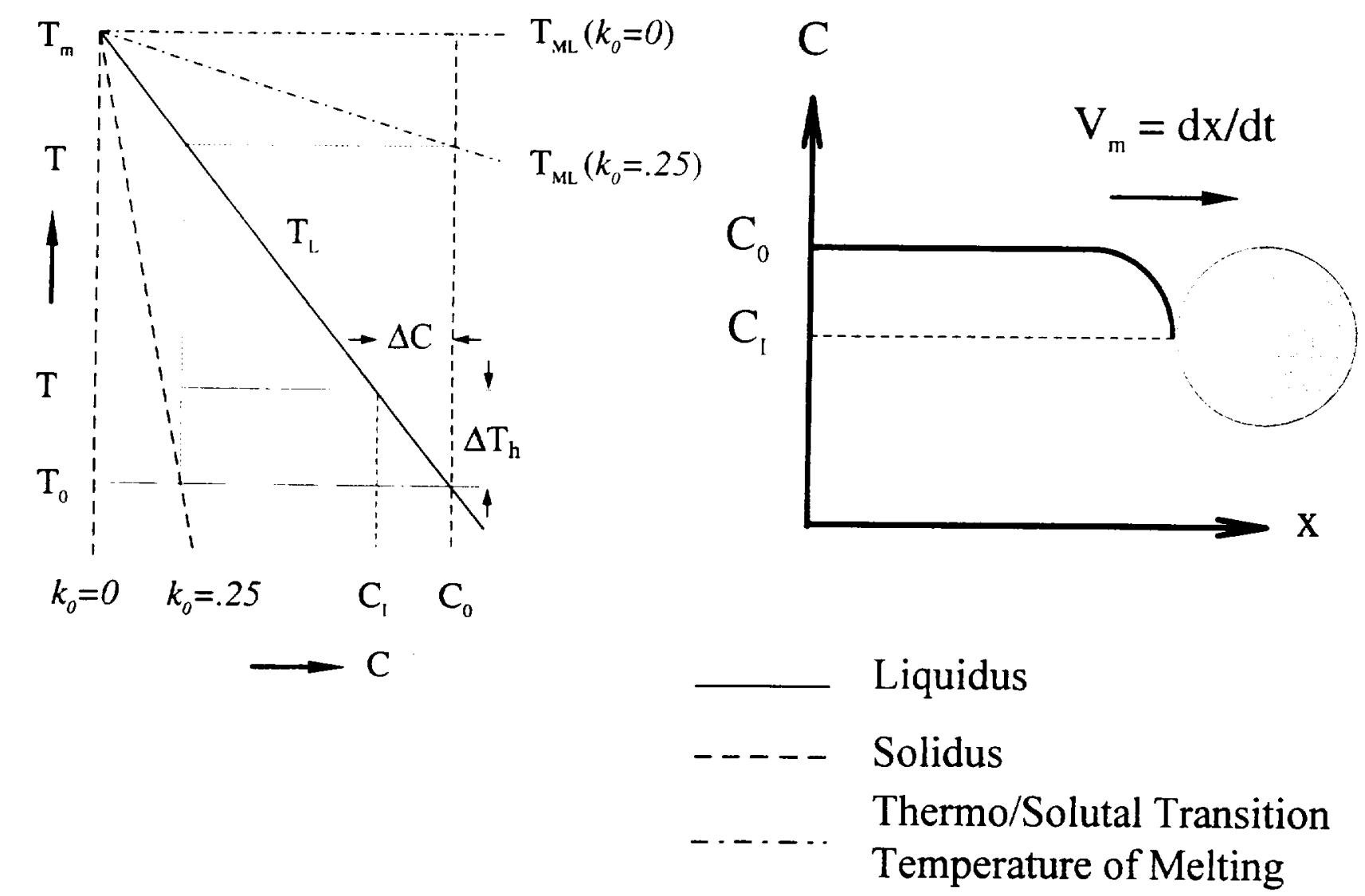

Fig. 7-Transition from solutally controlled to thermally controlled melting at a temperature, $T_{M L}$, for a case of zero solid solubility, $k_{0}=0$, and finite solid solubility, $k_{0}=0.25$. The solid line is the liquidus, and the bath composition is $C_{0}$, with freezing point $T_{1} 0$. At temperatures above $T_{00}$, up to $T_{M L}$ melting is controlled by solute diffusion and is slow; above $T_{M L}$, melting depends on heat flow and is rapid. The melting rate, $V_{m}$, at any temperature up
to $T_{M L}$ is proportional to $\Delta C=C_{0}-C_{l}$.

coefficient, and $\Delta r$ is some radial diffusion distance in the liquid, e.g., a boundary layer, $-\delta$. The rate is limited by solute/solvent diffusion and rises with the temperature as $C_{l}$ decreases and $\Delta C$ increases, until $T_{M L}$. At and above the melting point of the pure solvent, melting becomes heatflow dependent and reduces to a single component problem; composition adjustment then follows after or while the particle melts, which it does increasingly rapidly ( $c f \tau=$ $\left.\kappa / D_{L}\right)$ as

$$
V_{m} \Delta H=\frac{k\left(T-T_{m}\right)}{\Delta r^{\prime}}
$$

where $T$ is a bath temperature above the melting point, $\Delta H$ the latent heat, $k$ the thermal conductivity, and $\Delta r^{\prime}$ a larger characteristic distance for heat flow. In a more general case, with some solid solubility, e.g., $k_{0}=0.25$ (Figure 7), the same argument applies, but now the transition temperature from matter- to heat-controlled melting applies to a bath temperature for liquid $C_{0}$ at the corresponding solid melting composition at $k_{0} C_{0}$. The locus of such temperatures then follows a line

$$
T_{m \leq}=T_{m}-m_{L} k_{0} C
$$

It seems appropriate to call this a thermosolutal transition temperature for melting. Crude experiments with succinonitrile-water confirm changes in melting rates above $T_{m t}$ $\left(=T_{m}\right)$ by a factor of more than 30 times within less than $5 \mathrm{~K}$ above the melting point. ${ }^{[24]}$ For the $\mathrm{NH}_{4} \mathrm{Cl}-\mathrm{H}_{2} \mathrm{O}$ system, this transition is irrelevant, because the aqueous solutions are far below the solid melting point, but in foundry practice, in many metal systems, the pouring temperature may often exceed this melting transition temperature (for example, consider alloys such as Fe- 0.5 wt pct or Al-4 wt pct $\mathrm{Cu})$. This will mean that any metal fragments which are momentarily produced, as by local chill at mold surfaces, will have a very short lifetime indeed, unless the superheat is almost impracticably low. Reverting to the experimental aqueous system under consideration, approximate melting rates and hence life spans of the same order as are observed can be calculated for solute/solvent diffusion by taking a boundary layer thickness equal to the particle radius. These allow some estimates of expected particle densities to be made for given local superheats.

(c) Particle densities prevailing at a given ambient temperature must be a result of supply and survival, being a standard demographic problem of "birth rate" vs "death rate," where here, the former is the number of particles supplied by convection, $\hat{N}$ and the latter is the reciprocal of the melting life span, $\phi \mathrm{s}^{-1}$. The number of particles surviving in a given volume after a time, $t$, is then given by

$$
\begin{gathered}
\frac{d N_{1}}{d t}=\dot{N}-N_{1} \phi, \text { and with integration, } \\
N_{t}=\left(\frac{\dot{N}}{\phi}[1-\exp (-\phi t)]\right)
\end{gathered}
$$




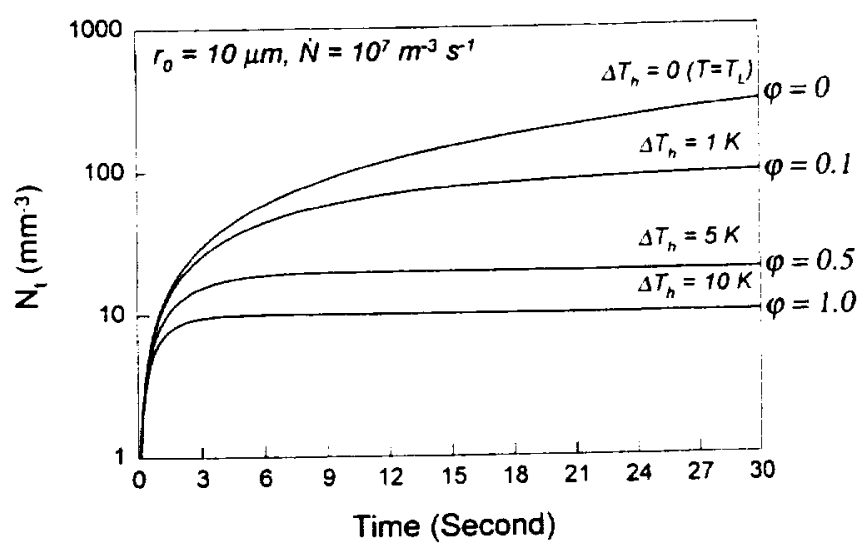

Fig. 8-Calculated particle population densities, $N_{n}$ after times, $t$, for a given rate of particle arrival or birth rate, $N$, and of particle radius, $r_{0}$, at melting or death rates, $\phi$, corresponding to various melt superheats, $\Delta T_{h}$, as in Fig. 7.

Experimentally, we do not yet have acceptable measurements of particle densities or of the quantities, $N$ and $\phi$, although an initial value for $N$ can be taken from numbers observed in fingers or plumes and estimates of $\phi$ are at least of the correct order. Thus, Figure 8 shows how particle densities should converge with time for assumed initial particle size $\left(r_{0}=10 \mu \mathrm{m}\right)$ and an observed supply rate of the order of $10^{7} \mathrm{~m}^{-3} \mathrm{~s}^{-1}$ at superheats from 1 to $10 \mathrm{~K}$. Convergence is more rapid as the melting rate or death rate increases, but the values are considered in general to be too high because many particles melt as they are transported through the hotter liquid before reaching some given volume element, as previously mentioned; i.e., $N$ is probably too high. Clearly, the situation of particular interest is with the small critical temperature range as the superheat falls below $\approx 1 \mathrm{~K}$, when nearly all ejected dendritic fragments begin to survive. Meaningful measurements of particle densities require very close correlation with accurate temperature measurements and local cooling rates, and the important transition range is narrow and almost abrupt.

\section{E. Growth and Sedimentation}

Below the freezing point, the situation changes abruptly, and from the foregoing (Figure 6(a)), this applies to much of the bulk liquid above the growth front. Initially spherical particles start to grow dendritically as soon as the undercooling exceeds that for curvature, $\Delta T_{r}$ (with $r=10 \mu \mathrm{m}$, $\sigma \approx 0.1 \mathrm{~J}^{-2}$ and the entropy of fusion, $\Delta S \approx 4.10^{8} \mathrm{~J}^{-3}$ $\mathrm{K}^{-1}$, and $\Delta T_{r}=2 \sigma / r \Delta S<10^{-4} \mathrm{~K}$ ). Initially, this will be unconstrained growth and will lead to recalescence within a short time, so that the bulk liquid rapidly becomes spatially isothermal. Simple calculations of unconstrained growth with solute rejection and with a cooling rate of $\approx 10^{-1} \mathrm{~K} \cdot \mathrm{s}^{-1}$, from a given number of intrinsic nuclei, indicate that recalescence will occur within $<10$ seconds. Recalescence is a consequence of growth and evolution of latent heat, so that within quite wide ranges of nucleation densities, the rate of recalescence does not actually differ very much, perhaps contrary to perceived wisdom. ${ }^{[29]}$ At this point, it is recognized that the modeling procedure begins to follow that which is generally used to fit with ther- mal analysis, cooling curve data ${ }^{25-28]}$ and becomes "standard." As noted previously, relatively close correlation with cooling curves is possible with a relatively wide range of nucleation sites.

In this range (of time and temperature), before impingement, the radial dendritic growth rate, $V_{r}$, follows:

$$
V_{r} \propto \Delta T^{n}
$$

where $n \approx 2$, and experimental data and analysis are available for this situation ${ }^{16,17,201}$ in single- and two-component systems.

Since the solid is invariably more dense than the liquid (by some 30 pct in $\mathrm{NH}_{4} \mathrm{Cl}-\mathrm{H}_{2} \mathrm{O}$ ), the growing equiaxed crystals sink according to Stokes' law, modified for an envelope of radius $r$, within which the solid fraction, $f_{s}$, is relatively low $(<5 \mathrm{pct})$. Terminal sedimentation velocities, $V_{T}$ are reached rapidly in the density ranges involved here, although the particles accelerate as they grow. Also, within the envelope which effectively encloses the dendritic crystal, the interdendritic liquid is slightly enriched in solute and is therefore marginally less dense than that of the surrounding liquid. However, although this fraction is large, the density difference, $\Delta \rho_{L / \%}$, is very small and the buoyancy contribution is negligible. The terminal velocity, $V_{T}$ may therefore be written

$$
V_{T}=\alpha g\left[f s \Delta \rho_{s / 2}+(1-f s) \Delta \rho_{L^{\prime \prime} / 2}\right] r^{2 / \eta}
$$

where $\alpha<2 / 9, g$ is the gravitational acceleration, $f_{s}$ is the solid fraction within the grain, $\Delta \rho_{S /}$ is the solid:bulk liquid density difference, and $\Delta \rho_{L / 2}$ is the difference between the liquids inside and outside the envelope. The value of $\alpha$ is $2 / 9$ for a sphere and the dendritic crystal outlines are approximately octahedral, but small shape changes make surprisingly little difference to Stokes' law, ${ }^{(30)}$ and in any case, crystals often rotate as they fall. Of more importance is the interaction between falling crystals, and as the separation between them decreases below about 10 diameters, the velocities decrease because of an effective upward displacement of the medium. ${ }^{[31]}$ It follows that as the particle density and size increase, the regime must change sharply as impingement begins, from one of falling particles through relatively stationary liquid to one of fluid flow through a stationary dendritic mesh. ${ }^{[37,381}$ Analysis of sedimentation rates in the $\mathrm{NH}_{4} \mathrm{Cl}-\mathrm{H}_{2} \mathrm{O}$ system gives good agreement with observations with $\alpha \approx 10^{-1}$ and $f_{s} \approx 0.02$ and has been scaled with some success to estimate rates and grain sizes in a continuous steel casting. ${ }^{[3]}$ Figure 9 summarizes this situation schematically, bringing the cycle of operations to a conclusion with the blocking of the columnar front from which fragment nuclei originated. Figure 10 is an example of this situation in the $\mathrm{NH}_{4} \mathrm{Cl}-\mathrm{H}_{2} \mathrm{O}$ system prior to the transition.

\section{F. Columnar-Equiaxed Transition}

The final step concerns a criterion for how many falling equiaxed crystals, and of what size(s), are necessary to block an upward growing, columnar dendritic front. Referring to Figure 10, a crude estimate of this necessary blocking density might be made as follows.

The columnar front is growing with a velocity, $V_{c}$, and with a primary dendritic spacing, $\lambda_{1}$, which is typically the 
Sedimentation-Columnar/Equiaxed Transition

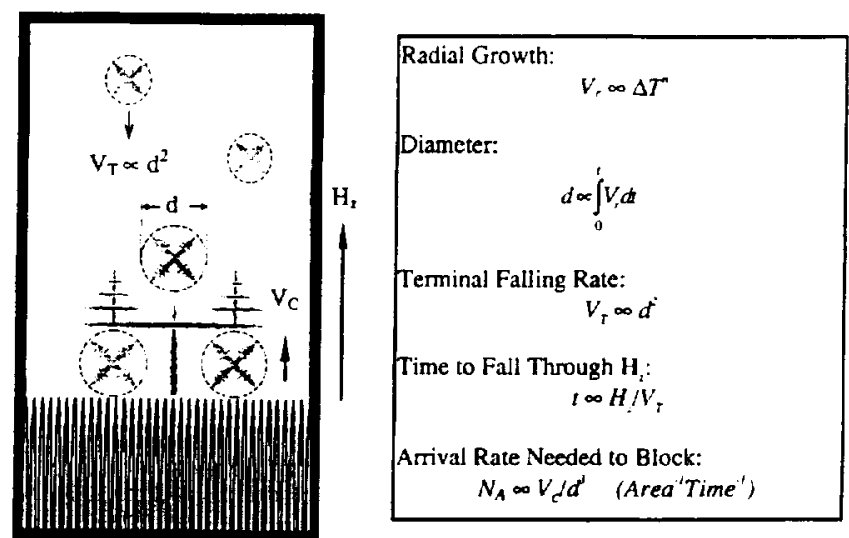

Fig. 9-Schernatic illustrating the growth of dendritic equiaxed crystals of diameters, $d$, falling at terminal velocities, $V_{r}$ toward the dendritic front which is growing upward at a velocity $V_{C}$.

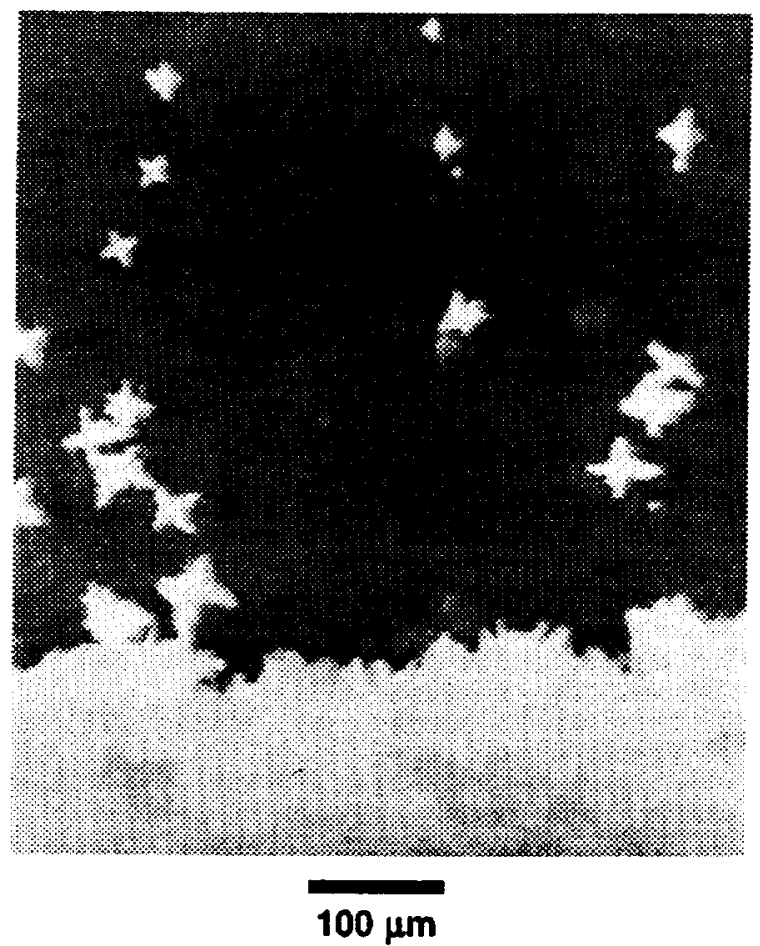

Fig. 10-Equiaxed crystals of $\mathrm{NH}_{4} \mathrm{Cl}$ falling onto the dendritic growth front in an aqueous casting.

same or less than the diameter, $d$, of falling crystals. The basic requirement to avoid blocking is that the columnar front be able to grow through a broken layer of obstacles in less time than it takes another equivalent layer to arrive, so that by dendrite branching and the development of tertiary arms, as indicated in Figure 9, the primary structure is largely retained. A simple criterion might be that the critical coverage of the surface should be $\approx 0.5$ in this time interval. Whatever the exact real fraction, the rate of arrival of obstacles per unit area, $N_{t}$, of diameter, $d$, will then be given by

$$
N_{A}=K \frac{V_{c}}{d^{3}}
$$

(where $K=1 / 4$ for 0.5 fraction). There are some obvious simplications here, notably, that all the falling crystals will not be the same size (coming from different heights) and that they also grow after they have arrived on the front (mostly in the vertical direction). A more comprehensive and sophisticated analysis ${ }^{1331}$ is to treat the arriving crystals in terms of nucleation and growth according to an Avrami formulation for extended volumes, and this actually does indicate a necessary arrival rate to effect $\approx 0.5$ coverage. For all these quantities, dimensions and rates can be measured and analyzed with some confidence; two points may be made. First, the blocking is obviously more effective by larger crystals, so that those originating only a little above the front are not as effective as those which have fallen further and had more time to grow (and also arrive faster). Therefore, there is a "most probable" level of origin for those equiaxed nuclei which are the most likely to block the front. Application of such analysis has been made for the model system and can be extrapolated with some confidence. ${ }^{[32.331}$ Second, unless there is a sudden "blizzard" of equiaxed crystals (which is often the case, however), the columnar-to-equiaxed transition is not abrupt but rather gradual, as stray equiaxed grains become incorporated into the general framework of the columnar array. Again, this is rather well recognized and observed in practice.

\section{SUMMARY AND CONCLUSIONS}

In the foregoing, we have emphasized the roll of thermosolutal convection as a mechanism to transport crystalline fragments within a casting, in particular, from within the mushy region of a columnar dendritic array into open, bulk liquid. The argument pursued throughout has been that such fragments are the most probable nuclei for equiaxed grains if no other substrates are deliberately introduced into the system.

Present schemes for the modeling of the grain structure of castings follow, with some sophistication, that of Oldfield, ${ }^{[23]}$ in which the question of origin of nuclei is avoided by use of expressions based on classical nucleation kinetics upon unspecified sites of variable potency. The modeling procedures which are then used, however elegant and elaborate they may be, ${ }^{[28,34,35]}$ must be classified as curve-fitting exercises to cooling curve data. This is not to detract from the demonstrable achievements of these models, but the present assertion is that sites can be identified in a physically realistic way and the separate steps to describe this alternative approach have been considered. This is not a simple undertaking and the necessary confirmation from experimental measurements is lacking for some parts of the operation, although the physical processes can be identified, albeit in a somewhat qualitative way.

Referring to the possible modeling sequence of Table II, the origin of crystal fragments is clearly identifiable (1), as is their transport (3), but the actual detachment kinetics of side arms from primary dendrite stalks (2) have yet to be clarified and experimentally confirmed. Likewise, working back from the end, reasonable physical understanding of the columnar blocking (6) and of the supply of equiaxed 
"obstacles" (5) is available, supported by acceptable observations and measurements. But, at present, the central connection, in terms of particle survival and particulate density (4), seems to be less tractable, although the kinetics of melting appear to be relatively straightforward, if poorly documented.

Inasmuch as a casting is a closed system, it would, in principle, be possible to write a computer program for stages (3) through (4), the number of particles escaping to that number needed to effect blocking, and to simply formalize the intermediate steps to yield a numerically compatible connection. Wang and Beckermann ${ }^{[36]}$ have outlined such a scheme to do this, but there is the danger that the physics of each individual, concurrent step will become obscure and that the model will then become no more physically predictive than those currently employed. This could easily become a more cumbersome and invidious exercise, even omitting the dendrite ripening and detachment processes and, as such, would offer no significant improvement over current models. However, we conclude that despite some current difficulties, solutions to the overall modeling sequences must be attainable, if only because the process is physically correct. It is relevant to note that in practice, the ranges of temperature which allow a sound casting to be made, combined with the physical properties of materials, are quite narrow, and in practice, without the addition of grain-refining substrates, the observed range of equiaxed grain sizes is limited to not much more than one order of magnitude, from around a hundred microns to a few millimeters. There is not much latitude here for meaningful results, although in terms of nucleation density, this size range does correspond to more than three orders of magnitude and is not as narrow as the grain size itself might imply.

Some comments are in order about the value of using transparent aqueous or organic analogues for metallic behavior and about extension from the vertical unidirectional casting geometry to other more general three-dimensional configurations.

There are two major inter-related differences between aqueous and metallic systems which require careful scaling because of the relative thermal conductivities and temperature ranges typically involved. The first of these is reflected in the Prandtl numbers (Table I) which, for metals, are some two or more orders of magnitude smaller than for the transparent materials. As previously remarked, this does not appear to alter the scale of convective patterns, but it profoundly affects the flow rates within them. Simultaneously, however, the higher thermal conductivity also allows heat flow to keep up with the rapid transport over similar distances (but shorter times) to those in the other systems, so that estimates of the distances within which convective plumes approach thermal equilibrium with surrounding liquid are quite similar, around $10 \mathrm{~mm}$.15] $^{[12}$ scales remain similar but the motion is dramatically accelerated, and there is some doubt as to whether a well-defined finger regime has time to develop in metals. In a base-chilled, lead alloy ingot, vertical segregation channels can be observed within a few millimeters of the chilled face ${ }^{(39)}$ and there is a very brief incubation period compared with aqueous systems. We conclude that in this respect, the transparent materials are valid analogues but in "slow motion."
Brief references were made to extrapolation from the vertical to other configurations, where thermosolutal and/or purely thermal convection lead to fluid flow across the dendritic growth front, i.e., normal to the heat flow direction. As noted, this blurs or obliterates distinction between finger and plume/channel patterns, leading to relatively long-range mixing throughout much of the solidification time. In terms of modeling, this more general or uniform mixing actually simplifies treatment of particle dispersion and survival, avoiding the necessity to consider local patterns and fluctuations. In fact, to complete a feasible modeling sequence, it may prove to be the only recourse, i.e., it may be necessary to ignore the distinction between finger and plume flow, in order to obtain a viable result. There are both gains and losses in this exercise.

Finally, it is appropriate to remark, again, that current modeling procedures are inevitably substantiated by comparisons with thermal analysis data by matching latent heat evolution to experimental cooling curves. The point to be reiterated is that such correlations are not a very sensitive function of the assumed or actual nucleation density, so that prediction of grain size by this method cannot be precise. This is the reason why some attempt should be made to physically describe where the nuclei originate.

\section{ACKNOWLEDGMENTS}

This work describes the focus of a research program which is supported by the National Science Foundation, Grant No. DMR-9206783, and the National Aeronautics and Space Administration, Grant No. NAG-3-1462.

\section{REFERENCES}

1. R. Rosenhain: J. Inst. Met., 1926, vol. 35, 282-85.

2. A Obno: Solidification: The Separation Theory and its Practical Applications, Springer-Verlag, New York, NY, 1987.

3. G.J. Davies: Solidification and Casting, Applied Science Publishing Co., London, 1973, ch. 6.

4. K.A. Jackson, J.D. Hunt, D.R. Uhlmann, and T.P. Seward: Trans. $T M S-A I M E, 1966$, vol. 236, pp. 149-60.

5. T.F. Bower and M.C. Flemings: Trans. TMS-AIME, 1967, vol. 239 , pp. 1620-27.

6. M.E. Glicksman and R.J. Schaefer: The Solidification of Metals, Iron and Steel Institute, London, 1968, Book 110, pp. 43-48.

7. J.S. Tumer. Buoyancy of Fluids, Cambridge University Press, Cambridge, United Kingdom, 1973, ch. 8.

8. J.S. Turner: Ann. Rev. Fluid Mech., 1985, vol. 17, pp. 11-44.

9. R.W. Schmitt: Ann. Rev. Fluid Mech., 1994, vol. 26, pp. 255-85.

10. S.M. Copley, A.F. Giamei, S.M. Johnson, and S.M. Hornbecker: Metall. Trans., 1970, vol. 1, 2193-2204.

11. R.J. McDonald and J.D. Hunt: Trans. TMS-AIME, 1969, vol. 245, pp. 1993-96.

12. A.K. Sample and A. Hellawell: Metall. Trans. B, 1982, vol. 13B, pp. 495-501.

13. A.K. Sample and A. Hellawell: Metall. Trans. A, 1984, vol. 15A, pp. 2163-73.

14. J.R Sarazin and A. Hellawell: Metall. Trans. A, 1988, vol. 19A, pp. 1861-71.

15. A. Hellawell, J.R. Sarazin, and R.S. Steube: Phil Trans. Roy. Soc. London, 1993, 345A, 507-44.

16. M.E. Glicksman and S.P. Marsh: in Handbook of Crustal Growth, D.T.J. Hurle, ed., North-Holland, Amsterdam. The Netherlands, 1993, vol. Ib, pp. 1075-1122.

17. B. Billia and R. Trivedi: in Handbook of Crystal Growth, D.T.J. Hurle, ed., North-Holland, Amsterdam, The Netheriands, 1993, vol. lb. pp. 899-1073.

18. S.Z. Lu and J.D. Hunt: J. Cryst. Growth, 1992, vol. 123, pp. 17-34. 
19. R.M. Sharp and A. Hellawell: J. Cryst. Growth, 1971, vol. 11, pp. $77-91$.

20. H. Muller-Krumbhaar and W. Kurz: in Materials and Technology: A Comprehensive Treatment, R.W. Cahn. P. Haasen, and E.J. Kramer, eds., VCH, New York, NY, 1991, vol. 5, pp. 553-62.

21. S.P. Marsh and M.E. Glicksman: Metall. Mater. Trans., 1996, vol. 27, pp. 557-67.

22. T. Sato, W. Kurz, and K. Ikawa: Trans. Jpn. Inst. Met., 1987, vol. 28. pp. 1012-21.

23. J.D. Hunt and S.Z. Lu: Metall. Mater. Trans., 1996, vol. 27A, pp. $611-23$.

24. Q. Han and A. Hellawell: Michigan Technological University, Houghton. MI, unpublished research, 1994.

25. W. Oldfield: Trans. ASM, 1966, vol. 59, pp. 945-58.

26. I. Maxwell and A. Heilawell: Acta Metall., 1975, vol. 23, pp. 229. 37 .

27. M. Rappaz and Ph. Thévoz: Solidification Processing, Institute of Metals, 1987, Book 421, 164-71.

28. D.D. Goettsch and J.A. Dantzig: Metall. Mater. Trans. A, 1994, vol. 25A, pp. 1063-79.

29. S.Z. Lu and A. Hellawell: $J$. Met., 1995, Feb., pp. 38-40.
30. G.H. Geiger and D.R. Poirier: Transport Phenomena in Metallurgy, Addison-Wesley Publishing Co., Reading, MA. 1973, ch. 3.

31. E. Bama and J. Mizrahi: Chem. Eng. J., 1973, vol. 5, pp. 171-89

32. J. Jang and A. Hellawell: Ironmaking Steelmaking, 1991, vol. 18, pp 267-83.

33. J.D. Hunt: J. Mater, Sci. Eng., 1984, vol. 65, pp. 75-100.

34. Ph. Thévoz, J.L. Desbiolles, and M. Rappaz: Metall. Trans. A, 1989 , vol. 20A, pp. 311-22.

35. Ch. Charbon, A. Jacot, and M. Rappaz: Acta Metall. Mater, 1994, vol. 42, pp. 3953-66.

36. C.Y. Wang and C. Beckermann: Metall. Trans. A, 1994, vol. 25A, pp. 1081.93 .

37. H.C. deGroh III, P.D. Weidman, R. Zakhem, S. Ahuja and C. Beckermann: Metall. Trans. B, 1993, vol. 24B, pp. 749-53.

38. C.Y. Wang, S. Ahuja, C. Beckermann, and H.C. deGroh III: Metall. Trans. B, 1994, vol. 25B, pp. 1-9.

39. J.R. Sarazin: Ph.D. Thesis, Michigan Technological University, Houghton, MI, 1992.

40. J.A. Spittle and D.M. Lloyd: Solidification and Casting of Mesals, The Metals Society, 1979, Book 192, pp. 15-20. 
